Service social

\title{
Protéger les jeunes et développer le pouvoir d'agir de leurs parents. Une analyse des pratiques d'implication parentale en centre jeunesse
}

\author{
Marie-Christine Saint-Jacques, Geneviève Lessard, Sylvie Drapeau et André \\ Beaudoin
}

Volume 47, numéro 3-4, 1998

Enfance : enjeux et réalités 2000

URI : https://id.erudit.org/iderudit/706796ar

DOI : https://doi.org/10.7202/706796ar

Aller au sommaire du numéro

Éditeur(s)

École de service social de l'Université Laval

ISSN

1708-1734 (numérique)

Découvrir la revue

Citer cet article

Saint-Jacques, M.-C., Lessard, G., Drapeau, S. \& Beaudoin, A. (1998). Protéger les jeunes et développer le pouvoir d'agir de leurs parents. Une analyse des pratiques d'implication parentale en centre jeunesse. Service social, 47(3-4),

77-114. https://doi.org/10.7202/706796ar

\section{Résumé de l'article}

Cet article rapporte les résultats d'une étude évaluative portant sur les pratiques d'implication parentale des intervenants sociaux du Centre jeunesse de Québec. La présente évaluation met l'accent sur les composantes et sur les processus relevant des pratiques qui contribuent à l'implication des parents dont un jeune reçoit des services en vertu de la Loi sur la protection de la jeunesse. Cet article porte, d'une part, sur les représentations qu'ont les intervenants de l'implication parentale et, d'autre part, sur les rôles qu'ils jouent en vue de favoriser cette implication. Ces résultats sont examinés à la lumière de la théorie sur l'empowerment des individus, qui constitue le cadre conceptuel de cette étude. 


\title{
Protéger les jeunes et développer le pouvoir d'agir de leurs parents Une analyse des pratiques d'implication parentale en centre jeunesse ${ }^{1}$
}

\author{
Marie-Christine SAINT-JACQUES \\ Geneviève LESSARD \\ Sylvie DRAPEAU \\ André BEAUDOIN
}

Cet article rapporte les résultats d'une étude évaluative portant sur les pratiques d'implication parentale des intervenants sociaux du Centre jeunesse de Québec. La présente évaluation met l'accent sur les composantes et sur les processus relevant des pratiques qui contribuent à l'implication des parents dont un jeune reçoit des services en vertu de la Loi sur la protection de la jeunesse. Cet article porte, d'une part, sur les représentations qu'ont les intervenants de l'implication parentale et, d'autre part, sur les rôles qu'ils jouent en vue de favoriser cette implication. Ces résultats sont examinés à la lumière de la théorie sur l'empowerment des individus, qui constitue le cadre conceptuel de cette étude.

In this paper, results of an evaluative study pertaining to parental involvement practices of social practicioners from Le Centre jeunesse de Québec are presented. The evaluation being described focuses on practice components and processes sustaining parental involvement of parents whose child is receiving services under Québec's Youth Protection Law. The representations that workers have, along with the various roles they adopt in order to encourage parental involvement, are discussed. The results will be examined in the light of empowerment theory that is the conceptual framework for this study.

1 Cette étude a été rendue possible grâce à une subvention accordée par le Fonds Richelieu de recherche sur l'enfance. On peut écrire à la première auteure à l'adresse suivante: Centre de recherche sur les services communautaires, 2446, Pav. Charles-de Koninck, Université Laval, Québec (Québec), Canada G1K 7P4. Courriel : marie-christine.saint-jacques@svs.ulaval.ca 
Quel type d'intervention est le plus efficace pour venir en aide aux jeunes et à leur famille suivis en protection de la jeunesse? Si la philosophie d'hier préconisait que les services sociaux se substituent aux parents "jugés incapables de prendre soin de leurs enfants ", les valeurs et les connaissances actuelles en la matière abondent en arguments fondés empiriquement voulant que la famille d'origine du jeune demeure, dans la grande majorité des situations, le milieu le plus propice à son développement et que toute interruption dans cette relation soit négative pour l'enfant (Leung, Cheung et Stevenson, 1994; Whittaker, 1990). On a ainsi assisté au passage d'une pratique sociale " paternaliste » à une pratique misant davantage sur les ressources et sur les capacités des clients à contrôler leur situation (Berg, 1996; Hegar et Hunzeker, 1988). Cette nouvelle vision de ce qui est "dans le meilleur intérêt de l'enfant » est venue changer considérablement le paysage des services de protection de la jeunesse, au Québec comme ailleurs. Pour s'en convaincre, il n'y a qu'à examiner les multiples projets d'intervention familiale visant le développement des capacités parentales, le maintien des enfants dans leur famille d'origine (family maintaining, family preservation), la réunification familiale quand le placement n'a pu être évité, etc. (Berg, 1996; Blythe, Jordana et Kelly, 1991; Boutin et Durning, 1994; Fraser, Pecora et Haapala, 1991; Laflamme, Lavoie, Desgagné et Marion, 1994; Paquet, 1997; Pecora, Wittaker et Maluccio, 1992; Rossi, 1992; Schatz et Bane, 1991; Schuerman, Rzepnicki et Littell, 1994; Simard, Vachon et Moisan, 1993; Whittaker et collab., 1990). Plusieurs pays, dont l'Australie et l'Angleterre, ont inclus dans leur loi de la protection de la jeunesse des articles prescrivant la participation des parents (Healy, 1998). Récemment, l'Association des centres jeunesse (1996) publiait un document - Vers une plus grande implication des parents et de leur jeune. Une réflexion à faire, des outils pour y parvenir - faisant la promotion de l'implication des parents et des jeunes dans l'intervention en protection de la jeunesse. La vision sous-tendant ce document est que, pour aider les jeunes, on ne peut se limiter à intervenir auprès d'eux. II apparaît en effet essentiel d'engager les parents dans le processus d'aide et d'ainsi maximiser les possibilités qu'ils jouent pleinement leur rôle auprès de leurs enfants. L'implication parentale devient donc un enjeu primordial de l'intervention en protection de la jeunesse, enjeu qui prend sou- 
vent l'allure d'un défi en raison des difficultés majeures que connaissent ces personnes et du contexte légal dans lequel doit s'établir la relation d'aide (Preston-Shoot, 1992).

Cet article présente les résultats d'une étude évaluative portant sur les pratiques d'implication parentale des intervenants sociaux du Centre jeunesse de Québec. À l'opposé des études évaluatives qui visent fréquemment à décrire les résultats d'une intervention (Rutman, 1982), la présente évaluation met l'accent sur les composantes et sur les processus qui contribuent à l'atteinte des résultats. En effet, si plusieurs recherches viennent démontrer qu'une intervention misant sur l'implication parentale permet d'obtenir des résultats plus positifs, peu d'études permettent de documenter ce qu'est un parent qui s'implique et ce que "font » les intervenants pour atteindre cet objectif. Dans cet article, nous examinerons, d'une part, les représentations qu'ont les intervenants de l'implication parentale et, d'autre part, les rôles qu'ils adoptent en vue de favoriser cette implication. Ces résultats sont examinés à la lumière de la théorie sur l'empowerment des individus, qui constitue le cadre conceptuel de cette étude.

Avant de passer à la présentation des résultats, nous présenterons le contexte particulier d'intervention qui préside à l'application de la Loi sur la protection de la jeunesse, de même que les aspects conceptuels et méthodologiques propres à cette étude. Nous exposerons en outre les principes fondamentaux orientant une intervention axée sur l'empowerment des individus.

\section{L'INTERVENTION DES CENTRES JEUNESSE QUÉBÉCOIS : UN CONTEXTE D'EXCEPTION POUR DES SITUATIONS EXTRÊMES}

Les centres jeunesse québécois ont la mission de rendre des services à la population en vertu des trois lois distinctes que sont la Loi sur la protection de la jeunesse, la Loi sur les jeunes contrevenants et la Loi sur les services de santé et les services sociaux. Dans la présente recherche, nous nous intéressons aux pratiques des intervenants dont le travail s'inscrit dans le cadre de la Loi sur la protection de la jeunesse. Cette loi a pour objectif d'assurer la protection de mineurs dont le développement ou la sécurité sont compromis. Les principaux problèmes traités sont la négligence, les troubles du comportement, l'abus physique, l'abus sexuel ainsi 
que l'abandon. Les situations portées à l'attention du Directeur de la protection de la jeunesse le sont par un mécanisme de signalement confidentiel. La négligence des enfants et les troubles du comportement chez les adolescents constituent, et de loin, le problème le plus fréquemment rencontré. Les cas signalés qui sont considérés comme recevables sont soumis à des intervenants chargés d'évaluer la situation et d'orienter vers la ressource la plus adéquate. Cette orientation pourra donner lieu à une prise en charge à l'intérieur même du centre jeunesse, cette prise en charge pouvant s'actualiser par des services de réadaptation (en externe ou en interne) ou de l'aide psychosociale. Dans le cadre de cette étude, nous nous intéressons aux pratiques des intervenants œuvrant dans ces différents secteurs, soit ceux de l'évaluationorientation, de l'aide psychosociale, de la réadaptation interne (placement en milieu institutionnel, de type centre d'accueil ou foyer de groupe) et de la réadaptation externe (réadaptation dans le milieu familial).

Par ailleurs, afin d'être en mesure de considérer l'univers dans lequel exercent les intervenants que nous avons rencontrés, il importe de souligner qu'au-delà du caractère exceptionnel des situations qui relèvent des actions des centres jeunesse les ressources humaines et financières dont disposent actuellement ces établissements ainsi que l'importance quantitative des cas signalés à l'attention des directeurs de la protection de la jeunesse contribuent à faire en sorte que seules les familles vivant des situations très difficiles arrivent à recevoir des services. De plus, bien que d'un point de vue légal certains services puissent être rendus en vertu d'une entente volontaire entre les partis, on s'entend généralement pour qualifier la pratique de ces intervenants d' « aide en contexte d'autorité ».

Pour espérer mettre un terme à une situation qui compromet la sécurité et le développement d'un enfant, on mise de plus en plus sur l'implication de tous les acteurs susceptibles de pouvoir améliorer cette situation, qu'il s'agisse du jeune lui-même, de ses parents, des réseaux de soutien de cette famille et des différents milieux dans lesquels celle-ci s'insère. Le souhait sous-jacent à cette tendance est que les premiers responsables du jeune, soit le jeune lui-même ainsi que ses parents, (re)prennent le contrôle de la situation. Or, il est par ailleurs aussi documenté (Boushel et 
Lebacq, 1992; Hegar et Hunzeker, 1988; Jenson et Whittaker, 1987; McCannell-Saulnier et Rowland, 1985) que ces personnes ont généralement peu de contrôle sur leur vie, qu'elles ont une faible estime d'elles-mêmes et de leurs capacités à jouer leur rôle de parents, qu'elles sont stigmatisées à maints égards en raison des difficultés qu'elles vivent (pauvreté, chômage, problèmes de santé mentale, toxicomanie, violence, échecs scolaires, etc.), qu'elles ont accès à peu de ressources, bref, qu'elles sont démunies, exclues et ont perdu confiance en leurs capacités. Callahan et Lumb (1995) rappellent d'ailleurs que peu de groupes de clients sont plus stigmatisés et blâmés que ceux qui sont accusés de négliger leurs enfants ou d'en abuser ou encore de ne pas les protéger contre les abus que leur font subir d'autres personnes. Toujours selon eux, cette stigmatisation serait d'autant plus difficile à modifier qu'elle est en quelque sorte institutionnalisée, légalisée par le contexte d'intervention qui prescrit une grande part du processus d'aide.

Si plusieurs travaux ont été consacrés à l'implication parentale, très peu ont tenté de documenter la façon dont les intervenants conçoivent cette implication et les rôles qu'ils privilégient dans le contexte aussi particulier qu'est celui de la protection de la jeunesse. La présente étude évaluative cherche à éclairer ces questions.

\section{CADRE CONCEPTUEL}

L'implication parentale signifie l'inclusion ou la participation active du père ou de la mère dans les activités, tâches, services et prises de décision tout au long de l'intervention des services de protection de la jeunesse. Ce concept se retrouve, à des intensités variables, dans différentes perspectives d'intervention, qu'elles soient dites écologiques, de préservation des familles, d'intervention de réseaux ou d'empowerment. Dans ce projet, l'implication parentale s'inscrit comme un des maillons essentiels d'un processus d'intervention qui vise l'empowerment de la clientèle. Une telle perspective entraîne un partage du pouvoir entre le client et l'intervenant, une analyse des iniquités, maintenues par la stigmatisation si importante des clientèles dans ce secteur, et une reconnaissance du caractère opprimant que peut jouer une organisation comme un centre jeunesse. Plusieurs auteurs soulignent 
qu'une importante source de cette oppression se trouve dans l'inégalité de pouvoir qui caractérise souvent les interactions des individus avec les structures et institutions sociales (Boushel et Lebacq, 1992; Callahan et Lumb, 1995; Cohen, 1998; Gerris et collab., 1998; Hegar et Hunzeker, 1988; Parsons, 1991; Perkins et Zimmerman, 1995). Cette inégalité de pouvoir contribue au développement de sentiments de culpabilité et d'impuissance de la clientèle face à son environnement. Cette clientèle se sent incapable de régler ses problèmes sans une aide extérieure, mais également méfiante à l'égard des organismes d'aide (Jenson et Whittaker, 1987; Parsons, 1991; Schatz et Bane, 1991).

Dans l'intervention en service social, la valeur d'autodétermination du client occupe une place centrale (Boushel et Lebacq, 1992; Parsons, 1991). Le but visé est de contrer l'oppression et d'aider la personne à reprendre le contrôle de sa situation. Une façon d'y parvenir consiste à impliquer le client dans le processus d'intervention (Hegar et Hunzeker, 1988; Koren et collab., 1992). Ce processus de reprise de pouvoir s'opère en relation avec l'environnement (Le Bossé et Lavallée, 1993).

La philosophie qui sous-tend l'empowerment était déjà présente à la fin du $19^{\mathrm{e}}$ siècle dans les settlements et les organisations de charité fondées, entre autres, par Richmond (Charpentier, 1997). Mais ce n'est qu'au milieu des années 1970 que le concept d'empowerment a été utilisé dans les écrits par Solomon (Hegar et Hunzeker, 1988; Schatz et Bane, 1991). Par la suite, de nombreux auteurs ont contribué à enrichir la théorie à ce sujet, dont Rappaport et Zimmerman. Ce concept réfère à un processus qui débute par la prise de conscience des conditions structurelles, qui conduit à l'acquisition d'un plus grand contrôle sur sa vie, qui à son tour entraîne une amélioration des sentiments d'estime de soi et d'efficacité personnelle et qui, éventuellement, se transforme en action collective visant une redistribution plus équitable du pouvoir et une transformation des structures opprimantes (Drolet, 1997; Jutras, 1996; Le Bossé et Lavallée, 1993; Parsons, 1991). Ainsi, l'empowerment est à la fois un processus et un résultat, une réalité objective et subjective (Drolet, 1997; Koren et collab., 1992; Parsons, 1991; Perkins et Zimmerman, 1995; Stark, 1992). En outre, l'empowerment se situe à plusieurs niveaux: individuel, interpersonnel et politique (Jutras, 1996; Koren et collab., 1992; 
Leon, 1999; Le Bossé, 1996), mais les auteurs ne s'entendent pas nécessairement entre eux sur les buts à atteindre afin de pouvoir statuer qu'il y a eu empowerment (voir Le Bossé et Lavallée, 1993; Callahan et Lumb, 1995). S'il faut éviter de personnaliser à l'extrême la notion d'empowerment de manière à mettre de côté l'objectif de changement social, l'empowerment psychologique n'en demeure pas moins un préalable au changement collectif (Le Bossé, 1996). Sans vouloir éluder le débat, nous adoptons la position voulant que, si l'empowerment à un niveau individuel ne permet pas de compléter l'ensemble de la démarche, il n'en constitue pas moins une étape essentielle. Pour cette raison, dans cette étude, la participation des parents n'est pas considérée comme un but, comme une fin en soi, mais plutôt comme un moyen permettant le développement de l'empowerment.

La définition de l'empowerment retenue dans la présente étude est empruntée à Le Bossé et Lavallée (1993) qui le décrivent comme « un processus par lequel une personne, qui se trouve dans des conditions de vie plus ou moins incapacitantes, développe, par l'intermédiaire d'actions concrètes, le sentiment qu'il lui est possible d'exercer un plus grand contrôle sur les aspects de sa réalité psychologique et sociale qui sont importants pour elle ou pour ses proches » (p. 17). Récemment, Le Bossé (1999) a travaillé à l'opérationnalisation du concept d'empowerment. Cette application, qualifiée de "développement du pouvoir d'agir », montre comment il est possible, dans l'action, de concilier les perspectives structurelles et individuelles qui président à une lecture des réalités sociales ainsi que des cibles d'intervention à privilégier. Nous aurons parfois recours à cette notion au cours de cet article.

\section{Les principes fondamentaux de l'intervention axée sur l'empowerment}

Il est possible d'identifier quatre principes fondamentaux sur lesquels repose une relation d'aide qui s'inscrit dans une perspective d'empowerment. Premièrement, la relation d'aide suppose la reconnaissance des forces, compétences et capacités des clients (Delgado-Gaitan, 1991; Gerris et collab., 1998; Hegar et Hunzeker, 1988; Hildebrant, 1996; Jutras, 1996; Leon, 1999; Le Bossé, 1996; McWhirter, 1998; Parsons, 1991; Perkins et Zimmerman, 1995; 
Stark, 1992; Webster-Stratton, 1998). Dans cette perspective, le client est considéré comme l'expert de sa situation et l'intervenant n'est plus le seul à posséder les ressources nécessaires à la modification de la situation (Gerris et collab., 1998; WebsterStratton, 1998). L'accent est mis sur la complémentarité des compétences de l'intervenant et du client; il s'agit de combiner les forces plutôt que d'offrir de l'aide (Callahan et Lumb, 1995; Drolet, 1997; Le Bossé, 1996; McWhirter, 1998; Striefel et collab., 1998). La relation intervenant-client doit donc être égalitaire et non hiérarchique.

La construction et le développement des réseaux de soutien permettent de mobiliser davantage les ressources du client et de l'intervenant. Ils permettent également d'élargir le travail de partenariat déjà entrepris entre l'intervenant et le client à une collaboration avec le réseau naturel de soutien de la personne ainsi qu'avec les autres organismes de la communauté (Cohen, 1998; Dickerson, 1998; Gerris et collab., 1998; Hegar et Hunzeker, 1988; Leon, 1999; McWhirter, 1998; Stark, 1992; Striefel et collab., 1998; Unger et Nelson, 1990; Webster-Stratton, 1998).

Deuxièmement, l'empowerment nécessite une réflexion critique par rapport aux inégalités et aux processus qui contribuent à maintenir la stigmatisation. Cette analyse devrait conduire au constat que ce sont les conditions structurelles et environnementales opprimantes qui gênent l'acquisition et le déploiement de compétences (Boushel et Lebacq, 1992; Callahan et Lumb, 1995; Delgado-Gaitan, 1991; Dickerson, 1998; Gerris et collab., 1998; Hegar et Hunzeker, 1988; Jutras, 1996; Koren et collab., 1992; Le Bossé, 1996; McWhirter, 1998; Parsons, 1991; Perkins et Zimmerman, 1995). Par conséquent, on ne doit pas blâmer les individus quand ils n'utilisent pas leurs compétences personnelles ou les ressources disponibles dans leur environnement. Par ailleurs, il ne s'agit pas non plus d'enlever aux individus la responsabilité qui leur incombe de prendre en main leur situation ou, pour les parents dans un contexte de protection de la jeunesse, d'assurer la sécurité et le développement de leur enfant. Pour faciliter cette réflexion critique, l'intervenant doit remplir les rôles d'observateur et d'analyste, c'est-à-dire être attentif aux processus sociaux qui contribuent à la stigmatisation, en aidant le client à en prendre conscience. 
Troisièmement, pour intervenir dans une perspective d'empowerment, il faut agir sur plusieurs plans, c'est-à-dire autant sur le plan individuel que collectif (Le Bossé, 1996; Stark, 1992). En fait, les actions concrètes occupent une place très importante dans l'intervention axée sur l'empowerment, puisque c'est par l'action que l'individu s'attribue la capacité d'influencer les événements importants de sa vie (Delgado-Gaitan, 1991; Dickerson, 1998; Gerris et collab., 1998). Plus concrètement, l'intervenant qui travaille dans une perspective d'empowerment aura le souci d'engager la personne activement dans le processus d'intervention. Cela signifie impliquer le client dans toutes les décisions qui le concernent, en lui fournissant l'information nécessaire pour qu'il puisse prendre des décisions éclairées par rapport à la planification de l'intervention et au choix des objectifs et des moyens (Cohen, 1998; Delgado-Gaitan, 1991; Dickerson, 1998; Drolet, 1997; Jutras, 1996; Le Bossé, 1996; Schatz et Bane, 1991; Striefel et collab., 1998; Webster-Stratton, 1998). L'intervenant se doit ensuite de respecter et d'appuyer la décision finale du client, même si celle-ci n'est pas conforme à l'orientation qu'il aurait souhaité donner à l'intervention ${ }^{2}$ (Boushel et Lebacq, 1992; Delgado-Gaitan, 1991; Drolet, 1997; Striefel et collab., 1998). Dans l'intervention axée sur l'empowerment, le client est au centre de l'action, alors que l'intervenant joue un rôle de facilitateur, d'entraîneur, d'accompagnateur et d'aidant dans le développement de l'empowerment de cette personne (Cohen, 1998; Drolet, 1997; Goyette, Marr et Lewicki, 1994; Le Bossé, 1996; Le Bossé et Lavallée, 1993). Sur le plan collectif, l'intervention comprend des activités de défense des droits et des mouvements sociaux qui visent à modifier les structures sociales de manière à améliorer les conditions objectives de vie des populations les plus défavorisées (Callahan et Lumb, 1995; Delgado-Gaitan, 1991; Dickerson, 1991; Drolet, 1997; Hegar et Hunzeker, 1988; Jutras, 1996;

2 On entrevoit ici une première limite à l'applicabilité de ce principe dans un contexte de protection de la jeunesse. Si la décision finale du parent compromet, aux yeux de l'intervenant, le développement ou la sécurité de l'enfant, le professionnel n'aura d'autre choix que d'amener le parent, à l'aide d'arguments de différents ordres (pouvant aller jusqu'à l'imposition par le pouvoir), à modifier son orientation. Mentionnons aussi que, pour Healy (1998), il ne s'agit pas d'une réelle limite puisque, de son point de vue, il faut reconnaître le caractère productif du pouvoir statutaire des intervenants en protection de la jeunesse. 
Le Bossé, 1996; Parsons, 1991; Schatz et Bane, 1991). Lors des actions collectives, le rôle du professionnel est d'agir comme un avocat envers les populations opprimées et comme un agent de changement (Drolet, 1997; Le Bossé et Lavallée, 1993).

Quatrièmement, puisque les problèmes sociaux sont complexes, les intervenants doivent utiliser une approche multidimensionnelle et différenciée selon les besoins de chaque client (Gerris et collab., 1998; Goyette et collab., 1994; Leon, 1999; Le Bossé, 1996). II n'existe donc pas de techniques et d'habiletés d'intervention spécifiques de l'approche axée sur l'empowerment. Par ailleurs, les intervenants qui s'inscrivent dans cette perspective utilisent une grande variété de méthodes d'intervention (Schatz et Bane, 1991).

Ces principes généraux conduisent à différencier l'intervention axée sur l'empowerment de l'intervention axée sur le contrôle. Cette distinction entre ces deux perspectives ainsi que l'impact de celles-ci sur le client sont clairement mis en évidence par Webster-Stratton (1998) :

The collaborative process has the multiple advantages of reducing attrition rates, increasing motivation and commitment, reducing resistance, increasing temporal and situational generalization, and giving parents and the therapist a joint stake in the outcome of the intervention. On the other hand, controlling or hierarchical modes of therapy, in which the trainer analyzes, interprets, and makes decisions for parents without incorporating their input, may result in a low level of commitment, dependency, low self-efficacy, and increased resistance, as well as resentment of professionals (p. 191).

Toutefois, McWhirter (1998, p. 24-25) soutient que, malgré les meilleures intentions, les services sociaux contribuent parfois à maintenir l'oppression. C'est le cas lorsqu'on présuppose la nécessité des services, que l'on encourage la dépendance et que l'on présume de l'équilibre du pouvoir. Les services deviennent encore plus opprimants si les intervenants ne sont pas habitués à l'auto-analyse critique de leurs privilèges et limites (Masson et Harrison, 1994; McWhirter, 1998). II est donc essentiel de reconnaître davantage les pouvoirs respectifs des intervenants et des 
clients et d'entretenir une volonté consciente de partager et de développer ce pouvoir (Cohen, 1998; Corby et collab., 1996; Gerris et collab., 1998). Ce risque de domination " inconsciente » serait cependant moins grand qu'on ne le pense. En effet, et contrairement à l'image fréquemment véhiculée au sujet des intervenants de la protection de la jeunesse : " there is considerable evidence to suggest that child protection workers often experience extreme difficulties in making negative judgments about the families with whom they work » (Healy, 1998, p. 906).

Dans le domaine de la protection de l'enfance, l'application intégrale de cette philosophie d'intervention s'avère plus difficile, puisqu'il s'agit d'un champ d'action encadré par des lois, ce qui oblige parfois les professionnels à prendre des décisions qui mettent de côté la participation des parents ${ }^{3}$ au profit du bien-être et du développement des enfants (Corby et collab., 1989; Striefel et collab., 1998). En fait, le point de rupture se situe au moment où les actions à poser pour développer l'empowerment chez les parents entrent en contradiction avec les actions à poser pour protéger l'enfant. Par ailleurs, plusieurs auteurs (Boushel et Lebacq, 1992; Callahan et Lumb, 1995; Corby, Millar et Young, 1989; Martone, Kemp et Pearson, 1989; Richardson, Galaway, Hudson, Nutter et Hill, 1995; Schatz et Bane, 1991; Striefel et collab., 1998) estiment qu'il est possible, dans un tel contexte, de mettre en place des interventions qui s'inscrivent dans une perspective d'empowerment. Ces interventions doivent toutefois respecter certaines conditions :

- Redonner aux parents la responsabilité de leur rôle parental, en leur permettant de prendre part aux décisions concernant l'enfant et en construisant à partir des stratégies positives déjà mises en place par ces familles.

- Informer les parents de leurs droits ainsi que du mandat (obligation de signaler les situations susceptibles de compromettre le développement de l'enfant)

3 À noter que, dans ce contexte, l'intervention axée sur l'empowerment concerne la relation de l'intervenant avec les parents plutôt qu'avec l'enfant qu'il protège (Boushel et Lebacq, 1992). 
et du fonctionnement des systèmes de protection afin d'éviter le pouvoir et le contrôle unilatéral.

- Offrir suffisamment de ressources aux parents en matière de temps d'intervention et de disponibilité.

- Respecter le rythme des familles, ne pas s'attendre à des pas de géant.

- Faciliter le changement non seulement des procédures d'intervention, mais aussi des valeurs et des croyances associées à une vision paternaliste des services sociaux. Le fait de disposer de politiques et de procédures de fonctionnement organisationnelles écrites facilite grandement le positionnement philosophique des intervenants en ce sens.

En somme, pour favoriser le développement positif des enfants, il apparaît de plus en plus essentiel de développer le pouvoir d'agir des parents en impliquant ces derniers dans l'intervention des services de la protection de la jeunesse. Dans la partie qui suit, nous chercherons à voir si la manière dont les praticiens conçoivent l'implication parentale et les rôles qu'ils adoptent pour susciter cette implication s'inscrivent dans une logique d'intervention qui vise le développement de l'empowerment des parents. Précisons que cette étude se centre particulièrement sur les pratiques (le faire). En effet, en analysant les propos des intervenants, nous cherchons moins à dégager l'opinion qu'ils ont à l'égard de cette philosophie de l'action qu'à examiner si ce qu'ils font pour impliquer les parents s'inscrit dans une telle philosophie.

\section{MÉTHOdologie}

Compte tenu de l'avancement des connaissances sur la question, la présente étude est de nature exploratoire et privilégie une méthodologie qualitative. La population à l'étude est constituée des intervenants du Centre jeunesse de Québec qui travaillent dans les secteurs de l'évaluation-orientation, de la réadaptation (interne et externe) et de l'intervention psychosociale. Les données ont été collectées à l'aide d'entrevues de groupe en s'appuyant sur les principes de la technique du focus group (Krueger, 1994; Simard, 1989), ce qui permet d'accroître la validité des résultats recueillis. Cette technique prévoit la présence 
d'un animateur et d'un observateur. Afin de recueillir les informations nécessaires à l'atteinte des objectifs, une grille d'entrevue de groupe a été élaborée et prétestée. Cette grille comprend une question d'introduction ("Pourriez-vous me décrire ce qu'est un parent impliqué dans un contexte de protection de la jeunesse, particulièrement dans votre secteur d'activité? »), suivie d'une tâche de transition où les participants doivent reconnaître et rapporter, par écrit, deux situations particulières en complétant les phrases suivantes: " J'ai senti que j'avais vraiment impliqué le parent dans l'intervention la fois où... ॥ et " II m'a été impossible d'impliquer le parent comme je l'aurais voulu la fois où... ». Sont ensuite abordées les questions clés (par exemple, "À partir des situations auxquelles vous avez pensé en faisant cet exercice, pourriez-vous me décrire ce que vous faites, en tant qu'intervenant(e), pour impliquer les parents dans l'intervention? " et des questions permettant de conclure l'entrevue de groupe (par exemple, "Si vous aviez une minute pour parlez de l'implication parentale en contexte de protection de la jeunesse, sur quel aspect vous attarderiez-vous? »). À la fin de l'entrevue, la personne chargée d'observer la rencontre livre au groupe un court résumé des idées centrales qui ont émergé de la discussion et recueille les réactions des participants (par exemple, « Est-ce que ce résumé vous apparaît complet? »).

Sept groupes de discussion qui réunissaient de 5 à 6 intervenants, sélectionnés au hasard, ont été tenus. Au total, 38 praticiens (26 femmes et 12 hommes) ont participé à ces groupes. La grande majorité d'entre eux sont âgés de plus de 35 ans. De manière générale, il s'agit d'intervenants d'expérience, puisqu'ils travaillent dans les services sociaux depuis plus de 16 ans, dont 13 ans en protection de la jeunesse. Plus du tiers possèdent une formation en travail social $(36,8 \%)$ ou en éducation spécialisée $(39,5 \%)$. Beaucoup plus d'intervenants possèdent une formation universitaire $(62,9 \%)$ plutôt que collégiale $(37,1 \%)$. Afin de recueillir les informations nécessaires à l'atteinte des objectifs, une grille d'entrevue de groupe a été élaborée et prétestée. Avec l'accord écrit des participants, le contenu des discussions a été enregistré et filmé. Par la suite, les propos des participants ont été retranscrits sous forme de comptes rendus in extenso (verbatim). Le visionnement de la bande vidéo a permis d'ajouter aux comptes rendus les expressions non verbales. Le matériel a été 
traité à l'aide de la méthode d'analyse de contenu thématique (Bardin, 1986; L'Écuyer, 1990). Un premier système de catégories a été élaboré. Le matériel de deux entrevues a été classifié par une personne, puis vérifié par une autre, ce qui a permis de parfaire le système de catégories. Par la suite, un seul codeur a finalisé l'analyse du matériel. Le logiciel Nud•lst a servi de support technique à cette analyse.

\section{LE CONCEPT D'IMPLICATION PARENTALE ET SON APPLICATION DANS LES PRATIQUES DES INTERVENANTS EN PROTECTION DE LA JEUNESSE}

Si a priori le concept d'implication parentale peut sembler univoque, l'analyse du discours des intervenants démontre que cette notion est loin de refléter une réalité homogène. En effet, dès le départ, une première distinction s'impose : selon le secteur d'activité où l'on travaille (intervention psychosociale ou réadaptation), l'implication parentale référera soit à l'implication des parents dans l'intervention, soit à l'implication des parents auprès du jeune. De plus, pour les intervenants chargés de la dimension réadaptation, le fait que le jeune soit placé dans un milieu substitut les amène à considérer comme étant un parent impliqué celui qui continue à agir auprès de son jeune, malgré un contexte qui limite les possibilités. En outre, ces mêmes personnes ont plutôt tendance à considérer leurs propres actions comme se limitant au jeune luimême, excluant par le fait même une représentation de parents impliqués dans l'intervention. Notons aussi que le concept d'implication parentale doit, selon les intervenants, posséder une certaine souplesse qui permet de prendre en considération les capacités et les ressources des parents.

Plusieurs intervenants se représentent le parent impliqué comme celui qui demeure le principal acteur dans la situation, qui se sent responsable du jeune et qui assume, malgré le contexte, son rôle de parent. Se représentant le parent dans leur propre catégorie, les intervenants estiment que le parent doit être considéré comme un expert et comme le principal intervenant, puisque c'est cette personne qui connaît le mieux le jeune. Ainsi, ces intervenants reconnaissent la compétence du parent et lui redonnent de la crédibilité, en lui faisant sentir qu'ils le considèrent comme un parent adéquat. Le fait de nommer les capacités des parents contribue à rehausser leur estime de soi et à réduire la stigmati- 
sation souvent vécue par ces parents auprès de qui l'on intervient parce qu'ils n'ont pas su protéger leur enfant adéquatement.

\begin{abstract}
Je pense que le parent peut devenir intervenant au même titre que l'intervenant du centre de réadaptation. Pourquoi un parent ne pourrait pas venir à un moment donné faire certaines interventions auprès de son jeune au centre de réadaptation? Comme prémisse, il faut se dire que le parent demeure toujours le premier intervenant de son enfant. (Gaston)
\end{abstract}

Moi je pense que pour impliquer les parents il faut leur donner de la crédibilité [approbation de Danielle], les valoriser dans leur rôle, c'est bien important [approbation de Danielle]. [...] J'ai donné beaucoup beaucoup de crédibilité à un père, alors qu'on avait toujours entendu dire qu'il était croche, qu'il était par en dessous... [approbation de Danielle] Et c'est vrai qu'il était comme ça, mais le fait de lui avoir donné de la crédibilité c'est comme si ça l'amenait à ouvrir [approbation de Danielle], à dire les vraies affaires plutôt que de toujours cacher les choses. [...] Pour moi, le lien de confiance que tu as à établir avec le parent, il est aussi important que celui à établir avec le jeune. Si tu veux que naisse une complicité, il faut que tu la développes et il faut que tu prennes le temps pour ça. (Marie)

Maintenant tu dis au parent, "tu es utile pour ton enfant, il a besoin de toi ». Alors tu travailles le lien d'appartenance, lui a besoin de peut-être redorer son blason comme parent, parce qu'il ne s'est jamais senti très très bon comme parent. Là tu dis, il a une utilité, vous pouvez faire quelque chose. Il est considéré, il va partager ce qu'il connaît de son fils et c'est ça que le parent a besoin d'entendre. (Pierre)

Les intervenants qui voient le parent comme le premier responsable du jeune partagent également avec lui le pouvoir décisionnel dans le choix des objectifs d'intervention et des stratégies d'action. Puisque le parent demeure la personne qui connaît le mieux l'enfant et la situation familiale, il doit, selon les intervenants, participer à la définition des moyens. L'implication dans les décisions lui permet de se réapproprier son pouvoir. 
Pour moi, un parent impliqué, c'est un parent qui reste parent, qui demeure responsable du jeune et avec qui on communique pour toutes les décisions à prendre. [...] au niveau des orientations, au niveau physique aussi, au niveau monétaire, comme un parent dans le fond. (Isabelle)

Dans une des situations que j'avais, c'était une grandmaman qui était très récalcitrante. [...] C'est elle qui avait la garde du petit-fils, la cour la lui avait donnée, et c'était pas facile d'approche. Parce que quand l'adolescent récriminait, on était à peu près sûr qu'on recevrait un téléphone et que la grand-mère tenait le même discours que le jeune. Si c'était difficile avec l'adolescent, j'étais sûr que c'était pour être dur avec la grand-mère. [...] Les moyens, on aurait pu les sortir tout seuls. Sauf que, en lui faisant élaborer elle des moyens, [...] c'est venu pas mal vite. [...] À partir du moment où on a quitté ce jeu-là pour dire "on a besoin de vous, qu'est-ce que vous voyez? », là on l'a impliquée. Avant je pense que c'était la partie peut-être où on avait pris le contrôle et, à partir du moment où on l'a partagé, on a évolué. (Pierre)

S'il s'avère important de reconnaître les compétences des parents, les informateurs rencontrés estiment qu'un parent réellement engagé dans l'intervention doit par ailleurs reconnaître le problème, reconnaître qu'il fait partie du problème et qu'en ce sens la solution ne passe pas uniquement par des changements chez le jeune. Cette reconnaissance, de l'avis des intervenants, n'est pas chose aisée pour les parents. En effet, la reconnaissance des problèmes familiaux et du rôle qu'ils ont pu jouer en tant que parents dans l'apparition de ces problèmes exige beaucoup d'humilité. La culpabilité ressentie par certains parents les empêche même de s'impliquer dans l'intervention, puisque cette dernière nécessite qu'ils remettent en question la façon d'exercer leur rôle parental. Sachant que le contexte d'autorité ne contribue nullement à faciliter cette remise en question, les intervenants ne s'attendent pas à ce que les problèmes soient tous reconnus dès le départ. En fait, il s'agit, pour les intervenants, de trouver un équilibre entre la stigmatisation associée à la clientèle suivie par les services de protection de la jeunesse et leurs tentatives d'amener le parent à reconnaître ses difficultés et sa responsabilité à l'égard de l'enfant. 
C'est dur quand même de reconnaître un problème officiellement. On peut vouloir être impliqué, mais avant il y a comme une démarche à faire, c'est d'être très humble pour dire, oui, je suis plus capable. J'imagine qu'un parent très impliqué, c'est un parent qui reconnaît qu'il a un problème, qui reconnaît qu'il a des faiblesses et qui veut de l'aide. (Micheline)

Par ailleurs, si la plupart des intervenants s'entendent sur le fait qu'un parent impliqué est ouvert et transparent, ces termes ne recouvrent pas la même signification pour tous. En réalité, certains intervenants se contentent d'un minimum d'ouverture au départ, en souhaitant que le parent puisse s'ouvrir davantage au fur et à mesure que la relation intervenant-client se développera. Selon eux, il apparaît tout à fait normal, dans un contexte d'intervention légale comme celui de la protection de la jeunesse, que les parents soient réticents à recevoir de l'aide. Pour ces intervenants, l'implication parentale est vue comme un " potentiel » à développer. D'autres intervenants estiment que l'ouverture à collaborer est présente seulement chez une partie des parents et qu'il est impossible d'impliquer un parent qui se montre réticent au départ. Ces intervenants se représentent l'implication comme une caractéristique «fixe », c'est-à-dire comme une disposition du parent qui est présente ou non, mais qu'il ne semble pas possible de développer par l'entremise de l'intervention. Le tableau suivant illustre, à l'aide d'extraits in extenso, les oppositions dans la façon dont les intervenants se représentent la transparence. 


\section{Tableau 1}

\section{Deux représentations différentes de la transparence dans l'implication parentale}

\section{L'implication parentale vue comme un « potentiel » à développer \\ Un parent qui s'implique c'est un parent qui accep- te au moins de me ren- contrer. Qui est prêt à regarder ce que je peux faire avec lui. C'est pas facile pour un parent de se voir envahir et puis [se faire] imposer des choses de l'extérieur. [...] C'est ce qu'on souhaite [que l'implication grandisse avec le temps]. En partant, ce que je souhai- te, c'est de travailler vrai- ment avec le parent. (Martin) \\ L'implication parentale vue comme une caractéristique " fixe » \\ C'est facile d'impliquer les parents quand les choses [projet de vie] sont claires. Si le parent dit " jamais plus tu vas revenir chez nous, moi ma capacité de t'aimer c'est quatre heures par deux semaines ", c'est facile de dire au parent " votre implication on va vous la demander quatre heures par deux semaines, "that's it that's all", le reste, bonsoir ". Mais en même temps, votre implication on va vous la limiter dans une petite boite et c'est comme ça qu'on va fonctionner. Mais si, d'un autre côté, il me dit "Pour l'année scolaire, à la fin des vacances, on ai- merait ça qu'il soit prêt à revenir à la maison ", l'implication va être plus facile. [...] La prémisse de base c'est qu'un parent veuille s'impliquer. II y a des parents qui se sentent menacés dans leur rôle, qui se sentent même jugés au début. (Ariane)}

Les intervenants mentionnent qu'un parent impliqué est capable d'être transparent par rapport à ce qui se passe à la maison, par rapport à ce que le jeune vit; il devient à la limite un complice dans l'intervention auprès de l'enfant. De son côté, l'intervenant doit aussi faire preuve de transparence, en expliquant les règles de fonctionnement du Centre, en donnant accès aux renseignements qui concernent sa situation, en l'informant des faits qui seront présentés en cour, en partageant les buts poursuivis. La transparence suppose donc une réciprocité dans la transmission 
d'informations entre le parent et l'intervenant et cette réciprocité favorise l'implication des parents qui se sentent davantage en confiance.

Deuxièmement, je pense que c'est important d'expliquer ce qu'on va faire. (Madeleine)

Parce qu'avant on allait au tribunal, le parent sait maintenant très clairement ce que je vais aller dire [approbation de Pierre]. Avant, on ne savait pas trop, on se ramassait au tribunal et on faisait comme éclater des bombes. Mais là, quand tu vas au tribunal, tu as préparé le parent avant. (Maude)

C'est de leur dire, déjà au départ, je vais aller vers vous, je vais vous demander votre opinion, mais je vais aussi vous donner la mienne. Et c'est une façon pour moi d'aller les chercher. (Yves)

La collaboration des parents dans le processus d'intervention apparaît un élément essentiel de la façon dont les intervenants se représentent l'implication parentale. Or, encore une fois, l'analyse du discours des intervenants fait ressortir des divergences de points de vue entre eux. Certains décrivent la collaboration comme un partenariat où le parent travaille dans le même sens que l'intervenant, en appliquant ce qui a été décidé avec l'intervenant. Cette conception suppose un partage du pouvoir entre l'intervenant et le client. Pour d'autres, un parent qui collabore est un parent qui se conforme aux normes édictées par l'intervenant et qui respecte les règles de fonctionnement de l'organisme. Le tableau suivant illustre, à l'aide des propos des répondants, ces deux conceptions différentes de la collaboration. 


\section{Tableau 2}

\section{Deux représentations différentes de la collaboration dans l'implication parentale}

\begin{tabular}{|c|c|}
\hline $\begin{array}{l}\text { L'implication parentale vue } \\
\text { comme un partenariat }\end{array}$ & $\begin{array}{l}\text { L'implication parentale vue } \\
\text { comme une soumission aux } \\
\text { normes et aux règles }\end{array}$ \\
\hline $\begin{array}{l}\text { Quand on parle de plan d'inter- } \\
\text { vention, il faut aussi acheter ce } \\
\text { que les parents nous disent et } \\
\text { c'est à partir de cela qu'on va } \\
\text { pouvoir les impliquer. (Gaétan) } \\
\text { Les parents, c'est important } \\
\text { qu'ils comprennent qu'ils ont un } \\
\text { rôle à jouer auprès de leur jeune, } \\
\text { que nous on en a un aussi et que } \\
\text { c'est ensemble qu'on peut y } \\
\text { arriver. Au départ, il faut que tu } \\
\text { établisses avec le parent qu'on } \\
\text { va faire une équipe ensemble, } \\
\text { qu'on est des partenaires. } \\
\text { Souvent, ça fait comme baisser } \\
\text { la tension du parent qui se sent } \\
\text { inadéquat ou coupable, " mon } \\
\text { jeune est là, je n'ai pas été } \\
\text { capable... ". C'est important que } \\
\text { les parents comprennent qu'ils } \\
\text { sont des partenaires, parce que } \\
\text { sinon les interventions seront } \\
\text { boycottées par le parent qui se } \\
\text { sent inefficace et inadéquat. } \\
\text { (Andrée) } \\
\text { Un parent impliqué c'est un } \\
\text { parent qui collabore, qui applique } \\
\text { ce qu'on a décidé ensemble. } \\
\text { (Johanne) }\end{array}$ & $\begin{array}{l}\text { On donne tout le temps des } \\
\text { photocopies du plan [d'inter- } \\
\text { vention], ils signent. Mais dans } \\
\text { l'action ils ne savent pas } \\
\text { comment ça se passe, ils ne } \\
\text { comprennent pas comment on } \\
\text { va l'appliquer dans le concret. II } \\
\text { faut tout le temps, même avec le } \\
\text { parent, le rappeler régulièrement. } \\
\text { (Marie-France) } \\
\text { Parce que [les parents séparés] } \\
\text { c'est des parents qui ne s'enten- } \\
\text { dent pas, ça fait qu'il faut diviser } \\
\text { à la lettre ce qu'ils ont le droit de } \\
\text { faire et ce qu'ils n'ont pas le droit } \\
\text { de faire. [...] II faut aussi dans le } \\
\text { fond qu'ils respectent leur } \\
\text { engagement, l'espèce de règle } \\
\text { de jeu que tout le monde s'est } \\
\text { donnée. (Ariane) }\end{array}$ \\
\hline
\end{tabular}

Pour plusieurs intervenants, l'implication parentale renvoie à l'action, à un comportement actif du parent qui travaille à l'atteinte des objectifs. Dans cette perspective, le parent impliqué est celui qui téléphone à l'intervenant pour connaître les interventions réali- 
sées auprès de son jeune, pour demander à rencontrer l'intervenant ou pour s'informer du fonctionnement en centre d'accueil dans les cas de placement. Un parent impliqué, c'est quelqu'un qui se présente aux rendez-vous fixés avec l'intervenant ou qui prévient ce dernier si un empêchement l'oblige à reporter le rendez-vous. Si certains intervenants croient que le parent devrait participer aux activités de la vie quotidienne de l'enfant, d'autres considèrent que l'implication n'exige pas nécessairement une présence physique mais que le parent peut très bien prendre part au processus d'intervention par des contacts téléphoniques, par exemple, dans la mesure où il est prêt à bâtir des stratégies d'action avec l'intervenant. En fait, les actions entreprises importent peu : elles peuvent prendre des formes très variées, du moment qu'elles conviennent au parent et lui permettent d'arriver à des résultats que lui-même jugera intéressants.

Un parent impliqué c'est un parent qui se prend en main : si tu lui fixes des rendez-vous, il est présent. S'il ne peut pas être là, au moins il t'en informe. (Maude)

[C'est] pas nécessairement [un parent qui accepte de venir]. Je pense que c'est un parent qui est prêt à faire des stratégies. Parce que peut-être que, physiquement, le parent il peut pas venir. On a eu un jeune [pour qui] l'intervention s'est faite au téléphone et très efficacement. Mais le parent est jamais venu en résidence. C'est pas un obligation, j'pense que c'est un moyen qu'on peut prendre. (Hélène)

Or, pour qu'un parent s'implique activement dans l'intervention, voire de façon proactive, il doit à la fois croire en la possibilité de changement, c'est-à-dire ne pas avoir abandonné l'idée qu'il peut améliorer la situation, et bénéficier du soutien de l'intervenant. En effet, les informateurs rencontrés dans cette étude ont souligné que, pour favoriser l'implication des parents, ils doivent d'abord s'impliquer eux-mêmes, en élaborant des stratégies d'intervention innovatrices ou en confiant des tâches aux parents afin de les mobiliser dans l'action.

C'est un parent qui va essayer des choses. Ce n'est pas le parent qui va dire " j'ai tout essayé, il n'y a plus rien à faire, occupez-vous-en vous autres ». (Richard) 
Mais là, il y avait toujours l'histoire du tribunal par en arrière et eux autres la résistance, méfiants comme vingt-cinq, le conjoint avec et tout ça. [...] ces parents-là, ils ne vont jamais aux rendez-vous, [...] ils ont toujours une raison eux autres. Tu sais, dans les milieux de négligence, le "char" a pas marché, la visite est arrivée, le frigidaire a sauté... [approbation générale] Alors, à un moment donné, la madame m'appelle et me dit: "Je ne pourrai pas aller à mon rendez-vous pour tatata ". Je lui ai dit: "Je vais y aller avec toi, je vais y aller te reconduire, on a du millage juste pour ça ». [...] C'était à des moments où la madame ne me parlait à peu près pas. Je vais la reconduire et elle me parle d'elle. Donc, ce que je veux dire, c'est qu'il y a des choses qu'il faut sortir du cadre de l'intervention comme telle [approbation générale] pour aller récupérer ça. (Gilles)

Je vais les mettre beaucoup en action. Ils vont écrire c'est quoi le plus gros malaise qu'il y a à travailler et combien de temps ils pensent qu'on va avoir à le travailler. [...] Moi je les fais beaucoup travailler comme ça. (Martin)

Un intervenant mentionne en ce sens l'efficacité de la collectivisation des problèmes individuels par l'intervention de groupe.

[...] que le parent ait à faire quelque chose, qu'il ait à poser des gestes, je pense que c'est de plus en plus vers ça que je m'en vais. L'important ce n'est pas juste de parler, de dire des choses, c'est de mettre quelque chose en application, c'est d'essayer quelque chose. [...] Une autre chose dont on n'a peut-être pas parlé, c'est la force des rencontres de groupe [approbation de Martin]. Les groupes de parents, entre autres, c'est très mobilisant et très renforçant aussi pour les parents [approbation de Martin]. C'est sûr que ce n'est pas un groupe pour se plaindre, c'est la même chose en individuel. J'essaye de sortir de ce guêpier-là, de recevoir des plaintes des gens qui se défoulent, parce que ce n'est pas mobilisant et ce n'est pas vers de l'action. J'essaie d'amener les gens à chercher des solutions, plutôt qu'à chercher à démontrer la difficulté de la situation. En groupe, je trouve que c'est merveilleux pour ça, que les 
gens se mobilisent pour une période donnée, d'une façon assez régulière. (Philippe)

Finalement, l'implication parentale dans un contexte de protection de la jeunesse requiert une grande souplesse de la part des intervenants. Puisque chaque famille possède ses particularités, les stratégies mises en place pour impliquer le parent doivent être différentes d'un dossier à l'autre. Cette attitude de souplesse s'inscrit dans une approche différenciée de l'intervention mise en œuvre dans une pratique visant le développement du " pouvoir d'agir " des individus. Par ailleurs, être souple signifie aussi entretenir des attentes réalistes par rapport à l'implication des parents en regard de leurs capacités.

Moi, c'est certain, je n'essaie pas d'avoir un modèle unique, parce que chaque situation est différente. $\dot{A}$ un moment donné, tu peux faire une action avec une famille, ça va bien, mais avec l'autre famille, ça ne sera pas approprié. (Martin)

Souvent, ce que je vais faire à la première rencontre, c'est que j'ai un plan d'intervention que je voudrais faire, mais je vais peut-être faire un objectif, je ne ferai peutêtre pas tout, parce que ça va être important de la connaître cette personne-là. [...] Je ne peux pas lui demander beaucoup si elle est très carencée, si elle a un vécu lourd... Et, par la suite, je vais peut-être bien aborder un premier objectif qui va dépendre de ses capacités, si elle est collaborante, si elle est encore méfiante, mais ce que je vais lui demander, c'est une petite chose. [...] Partir de ses points forts à elle, l'amener à cheminer et non pas l'obliger ou lui demander des choses irréalistes. (Madeleine)

\section{Les différents rôles que jouent les intervenants en vue d'impliquer les parents}

Dans un deuxième temps, l'analyse du discours des participants a conduit à l'identification des différents rôles que peuvent assumer les intervenants en vue de favoriser l'implication des parents. On observe que ces rôles sont étroitement liés aux conceptions de l'implication parentale. Par exemple, un intervenant qui définit cette implication par la primauté de la responsabilité du parent vis- 
à-vis de son jeune sera plus porté à se voir comme quelqu'un qui offre un soutien ponctuel à un adulte éprouvant une difficulté temporaire dans l'accomplissement de son rôle parental. Par contre, un intervenant qui considère que le parent impliqué est celui qui se conforme aux règles édictées sera davantage porté à jouer un rôle axé sur le contrôle et l'autorité. L'analyse des propos des répondants a ainsi fait ressortir quatre rôles principaux qu'adoptent les intervenants afin de favoriser l'implication parentale. Ces catégories ne sont pas exclusives, un intervenant pouvant assumer plus d'un rôle.

\section{L'intervenant " de passage »}

L'intervenant « de passage » définit principalement son rôle comme celui d'une personne qui offre un soutien ponctuel au jeune et à sa famille. Considérant les parents comme les premiers responsables de leur enfant et la situation-problème comme leur appartenant, cet intervenant évitera de se substituer à eux, même dans les situations de placement. II se perçoit comme un outil, comme une personne qui donne un service complémentaire. L'intervenant " de passage » étant particulièrement conscient du caractère temporaire de sa présence dans la situation, il a tendance à graduer son intervention en étant très présent au début de la prise en charge et en se retirant progressivement au fur et à mesure que la situation se dénoue. Même s'il tient à ne pas se substituer au parent, il peut toutefois le remplacer temporairement dans les aspects moins agréables de son rôle, comme celui de la discipline. Le but d'une telle action est d'améliorer la qualité de la relation parent-enfant, ce qui permettra par la suite de transférer progressivement au parent l'ensemble des responsabilités qui lui reviennent.

On demande au parent de se mêler, d'être plus proche de son gars. C'est à nous de les guider et d'aller les chercher par des contacts fréquents. Là il s'est passé telle affaire... autant dans le positif que dans le négatif. II faut les apprivoiser, les ramener près de leur jeune. Je ne serai pas toujours là et l'équipe ne sera pas toujours là. C'est vous qui devrez reprendre le rôle, c'est votre rôle. (Sylvaine) 
Je vais essayer autant que possible de pas faire à la place du parent, de lui donner des outils de travail, mais de ne pas gérer... Je ne la gérerai pas la crise, je vais essayer de lui faire voir des choses, de l'amener à [...]. S'il y a un gros conflit entre les parents et le jeune, je vais essayer de les amener à se parler, à communiquer. (Johanne)

Ici [au foyer d'accueil], c'est vraiment un endroit de transition. Je pense que l'intervention, même sur le plancher, se fait régulièrement de cette façon-là, qu'on n'est pas les gens qui vont s'occuper de lui tout le temps, ça va être son parent. [...] Je vais parler de ma situation. C'est une situation où le jeune s'en allait en appartement et le parent n'était pas du tout en contact, il ne voulait rien savoir. Et juste de l'appeler pour dire: "Vous restez son parent et c'est important que vous restiez là ", ça a amené, à fin du placement, le jeune à dire: "Je veux retourner chez nous." Ça a pris un mois, un mois et demi, comme implication chez eux et il est encore là. Ça ne se dessinait pas du tout qu'il retournerait chez eux. Mais en impliquant le parent quand même, même s'il ne retournait pas chez eux, ça a fait que le jeune a dit : " Ah, c'est drôle, mon parent s'implique. " II est retourné chez eux et les parents étaient très d'accord. (Hélène)

Quand le jeune dépassait, on le ramenait [...] on faisait le méchant loup. On jouait le rôle de la limite. Après ça, on a pu se retirer, la limite, c'est les parents qui la mettaient. Parce qu'ils sont toujours en conflit, ils sont toujours en discipline. [...] Ce que tu peux faire, c'est tout simplement établir des plages, on appelle ça faire des dépôts. Chaque fois que vous allez avoir une petite discussion le "fun », une petite activité le "fun", vous venez de mettre de l'argent en banque. Ainsi, quand viendra le temps de faire un retrait, si vous avez un petit fond, ça sera plus facile. (Isabelle)

Selon les intervenants, il est important d'y aller graduellement dans le transfert des responsabilités. Cela contribue à faire vivre au parent des réussites dans l'accomplissement de son rôle et, par conséquent, à augmenter chez lui l'espoir du changement. 
On se situe quand même dans le temps. Je pense que ça ne vient pas nécessairement... C'est une démarche évolutive. Elle [la mère] commence comme ça, elle est très encadrée, à un moment donné si tu as une implication, des changements ou la possibilité d'entrevoir une collaboration, tu vas aller vers le client et tu vas lui en donner de plus en plus de place, parce que tu vas peutêtre lui redonner ses enfants assez rapidement avec de l'aide. (Madeleine)

\section{L'intervenant « entraîneur »}

À l'image d'un entraîneur, ce type d'intervenant affirme que son rôle consiste à encadrer, à guider et à enseigner. II se perçoit comme la personne qui a la meilleure vision d'ensemble de la situation-problème en raison de sa position extérieure à la famille. L'intervenant " entraîneur » estime qu'il a la connaissance de ce qu'il faut pour gagner la partie, pour régler la situation. II ne peut toutefois pas remplacer les joueurs. Ainsi, à l'instar de l'intervenant "de passage ", il évitera de se substituer aux parents dans l'accomplissement de ses rôles et veillera à ce que ces derniers mettent eux-mêmes en application les stratégies qu'ils ont choisies ensemble en vue d'atteindre les objectifs visés et de reprendre le contrôle de leur situation.

On fait beaucoup d'enseignement, parce que les parents, quand ils arrivent là, ils sont démunis, ils ont besoin de pistes de solution. Donc, quand ils sentent que l'intervenant sait où il s'en va, ça les rassure aussi. Moi l'exemple que j'avais écrit, c'est un peu ça, c'est un couple qui était très instruit, et des gens très à l'aise, surprotecteurs, qui avaient jamais mis aucune limite à leur enfant. [...] Lui il pétait des crises terribles... comme un petit enfant. Ça fait que, ils ont accepté d'appliquer trois consignes très simples et, à partir de là, l'enfant s'est conformé. C'est des parents qui cherchaient toutes des solutions. C'était la première fois qu'ils apprenaient c'était quoi une limite. (Isabelle)

Quand on dit "faire avec ", c'est d'être là, de le faire avec eux autres, de les accompagner, on parle en termes de modeler, "coaching ". Par la suite, les parents ont quelques moyens et ils sont capables de le faire. (Gaétan) 
Et à un moment donné je regarde une situation, la jeune consommait toutes les fins de semaine chez elle, de la boisson, [...] les parents m'ont appelée, on a discuté ensemble comment eux voyaient ça et c'est eux autres qui ont décidé, c'est pas moi qui ai dit, bon, elle sortira plus, ou elle va rester au foyer. [...] Mais ils ont à composer des trucs aussi. Souvent les parents savent même pas qu'il y a des conséquences. Tu te dis ça n'a pas de bon sens, mais il leur manquait rien que ça et, à partir de là, ça va. (Caroline)

Il y a une maman qui n'arrivait pas à organiser la période d'étude, les devoirs n'étaient jamais faits. À partir $d u$ moment où moi je l'ai fait, pendant une ou deux semaines avec les deux enfants à la table et dans la position... À un moment donné, je suis arrivée et les chaises étaient déjà placées pour faire l'étude et elle disait "je n'ai plus de problème " et elle n'en avait plus de problème. Elle avait développé un mécanisme pour l'étude. Donc, pour elle, tous ses problèmes étaient réglés. Des fois, c'est par le « modeling ». (Évelyne)

\section{L'intervenant « médiateur »}

L'intervenant " médiateur » considère que son rôle est de favoriser la communication parent-enfant et de travailler à la reconstruction de la relation entre eux. Ce type d'intervenant accorde beaucoup d'importance aux liens d'attachement parent-enfant. II est particulièrement attentif, dans ses actions visant à impliquer les parents, aux réponses de ces derniers. En sollicitant l'implication du parent on peut aussi en arriver au constat qu'il est vain de vouloir continuer dans cette voie. En effet, si cet exercice conduit à déceler un rejet affectif du parent envers l'enfant, l'intervenant sera en mesure de mieux orienter son action vers une clarification du projet de vie du jeune, dont la nature variera en fonction de l'âge de celui-ci. Pour les plus jeunes, on peut entrevoir un placement à plus long terme et, pour ceux qui atteindront la majorité sous peu, les préparer à aller vivre en appartement supervisé.

Quand tu assois le père face au fils et que tu fais dire au fils " regarde, moi j'aimerais ça faire telle chose avec toi, qu'est-ce que tu fais? ». Ce n'est pas toujours facile, 
mais je pense qu'à un moment donné il faut, en termes d'intervention, en arriver là. Je pense qu'il faut être capable de lire un peu. II y a des parents qui se désistent, qui abandonnent, qui rejettent. À ce moment-là, l'intervention va prendre une direction autre que celle de l'implication. C'est peut-être aller vers une clarification de placement, de projet de vie. Donc il faut savoir jauger la situation, c'est important. (Gaston)

[...] le père disait, moi je m'attends à un peu plus de conversation de la part de ma fille, sauf que la fille me disait, mon père, il ne s'ouvre jamais, lui. J'avais à faire le pont entre les deux: "Vous savez, votre fille trouve ça difficile des fois, elle a l'impression que c'est souvent elle qui doit amener les choses, elle aurait le goût que vous vous préoccupiez d'elle, que ça vienne de vous. " La fin de semaine d'après, ils se sont assis et ont discuté. (Caroline)

\section{L'intervenant « figure d'autorité »}

L'intervenant qui incarne la "figure d'autorité » considère que son rôle consiste à contrôler et à " confronter » les parents de manière à ce qu'ils se conforment aux attentes et aux règles édictées par le praticien ou, plus généralement, par l'établissement. II se place dans une position de pouvoir face aux parents, notamment en prenant la majorité des décisions qui concernent le jeune seul. Ces intervenants tentent d'impliquer les parents dans leur intervention plutôt qu'auprès du jeune.

Une façon d'impliquer les deux parents [séparés] c'est, si la mésentente règne, notre mandat à nous autres en réadaptation n'est pas de faire régner la paix dans leur... C'est plutôt de travailler avec le jeune mais... Mais là travailler avec un jeune qui tire les ficelles d'un bord pis de l'autre, pis que la mère qui en profite pour "bitcher" le père... Donc, on a mis ça clair: "Vous, vous avez droit à cette partie-là et si vous voulez vous impliquer pour que votre jeune aille mieux, eh bien il va falloir que vous respectiez ça. » (Ariane)

On les a comme confrontés au problème parce qu'ils n'admettaient jamais leurs problèmes. [...] Et ça c'était un gros hic aussi, parce que, ce qu'ils voulaient, c'était le 
retour de la jeune, mais on ne l'autorisait pas, tant qu'ils ne respectaient pas nos conditions. Je pense qu'au niveau de l'admission du problème, ça peut être de confronter, des fois t'as pas le choix, tu leur dis, il y a ce problème-là, il faut que vous l'admettiez. (Léa)

Évidemment, il peut arriver qu'un intervenant en protection de la jeunesse se retrouve dans l'obligation d'assumer son rôle d'autorité parce que la situation d'un enfant réclame l'adoption d'une telle attitude. Cécile, toutefois, souligne l'importance de savoir discerner quand il convient de jouer ce rôle, car, selon elle, dans plusieurs situations, y compris une problématique de négligence, il est possible d'impliquer le parent sans être autoritaire ou contrôlant.

Mais ce n'est pas toujours dans toutes les situations. Il y a des situations où tu n'as pas à l'utiliser cette autoritélà. Il y a des degrés là-dedans et il faut savoir comment utiliser l'autorité quand c'est nécessaire de l'utiliser. (Cécile)

\section{DISCUSSION}

L'objectif de cet article était de décrire les représentations qu'ont les intervenants sociaux de la notion d'implication parentale dans un contexte de protection de la jeunesse ainsi que les rôles qu'ils assument en vue de favoriser cette implication. Nous tenterons maintenant de comprendre si les pratiques des intervenants s'inscrivent dans une perspective favorisant l'empowerment. Plusieurs des informateurs rencontrés dans cette étude se représentent le parent comme un acteur compétent qui peut agir auprès de son enfant au même titre que l'intervenant et qui joue un rôle clé dans la résolution du problème, puisqu'il demeure la personne qui possède la meilleure connaissance du jeune. Ce parent vit toutefois dans un contexte particulièrement difficile qui l'empêche d'exercer, sans appuis extérieurs, son rôle de manière à assurer la sécurité et le développement de son enfant. Dans cette perspective, les intervenants voient leur travail comme une aide ponctuelle qui ne peut avoir de réel impact sans l'investissement du parent. Ainsi, l'action de ces intervenants s'appuie sur l'un des principes fondamentaux d'une pratique axée sur l'empowerment, soit la reconnaissance et la complémentarité 
des compétences du client et de l'intervenant (Callahan et Lumb, 1995; Drolet, 1997; Gerris et collab., 1998; Le Bossé, 1996; McWhirter, 1998; Striefel et collab., 1998; Webster-Stratton, 1998).

Par contre, si certains intervenants conçoivent l'implication des parents comme un potentiel à développer, d'autres se la représentent comme une caractéristique fixe. Les premiers respectent davantage la philosophie de l'empowerment, puisqu'ils se centrent sur les capacités de changement des personnes plutôt que sur leurs déficits (Delgado-Gaitan, 1991; Gerris et collab., 1998; Hegar et Hunzeker, 1988; Hildebrant, 1996; Jutras, 1996; Leon, 1999; Le Bossé, 1996; McWhirter, 1998; Parsons, 1991; Perkins et Zimmerman, 1995; Stark, 1992; Webster-Stratton, 1998), respectent le rythme de changement et conçoivent l'individu comme un être dont le comportement résulte de forces systémiques plutôt qu'individuelles (Boushel et Lebacq, 1992; Callahan et Lumb, 1995; Delgado-Gaitan, 1991; Dickerson, 1998; Gerris et collab., 1998; Hegar et Hunzeker, 1988; Jutras, 1996; Koren et collab., 1992; Le Bossé, 1996; McWhirter, 1998; Parsons, 1991; Perkins et Zimmerman, 1995).

D'autre part, les intervenants qui voient le parent comme le principal responsable de son enfant estiment que celui-ci devrait être impliqué dans les décisions qui le concernent. L'implication du parent dans toutes les décisions se rapportant à l'enfant permet au premier de ne pas se sentir dépossédé de son rôle, de ses responsabilités, bref, de son pouvoir (Le Bossé, 1996). D'ailleurs, une pratique orientée vers l'empowerment établit que le client est la personne la mieux placée pour définir le problème et les solutions à ce problème (Cohen, 1998; Delgado-Gaitan, 1991; Dickerson, 1998; Drolet, 1997; Jutras, 1996; Le Bossé, 1996; Schatz et Bane, 1991; Striefel et collab., 1998; Webster-Stratton, 1998). La pratique de la plupart des intervenants s'inscrit dans cette vision du parent perçu comme un partenaire qu'il est nécessaire de consulter et avec qui il faut travailler très étroitement, puisqu'il s'agit de sa situation, de ses enfants, de sa vie.

De manière plus singulière, des intervenants sont d'avis que le parent doit d'abord et avant tout se conformer aux normes et procédures de fonctionnement organisationnelles. Ce client a été en quelque sorte jugé incompétent et l'organisation, légitimée par 
la Loi sur la protection de la jeunesse, lui retire une partie de son pouvoir d'agir en raison de ses incompétences. La solution aux difficultés vécues passe donc par le contrôle du pouvoir d'agir de ce client.

L'importance que les intervenants accordent à l'implication active, voire proactive des parents, notamment par la mise en œuvre de stratégies mobilisantes, témoigne aussi de l'inscription de leur pratique dans une perspective d'empowerment. Des auteurs mentionnent d'ailleurs que c'est à travers les actions entreprises par l'individu lui-même que celui-ci acquiert le sentiment qu'il a le pouvoir d'agir et d'effectuer les changements nécessaires à l'amélioration de sa situation (Delgado-Gaitan, 1991; Dickerson, 1998; Gerris et collab., 1998; Le Bossé, 1996).

Nous fondant sur les résultats de la présente étude, nous pouvons suggérer que la conception que se font les intervenants de l'implication parentale s'inscrit, pour certains, dans une démarche visant plutôt l'empowerment des individus et, pour d'autres, dans une action visant davantage le contrôle des personnes. Entre ces deux extrêmes, se trouvent les intervenants qui conservent un certain pouvoir du fait qu'ils possèdent plus de connaissances et d'expérience que le parent pour déterminer ce qui est le mieux pour l'enfant, mais dont l'objectif est d'enseigner au client et de le guider dans l'accomplissement de son rôle parental. Les résultats de cette recherche corroborent donc ceux de Cohen (1998) qui mettent également en évidence la présence de ces trois positions chez les intervenants sociaux, selon le degré de partage du pouvoir dans la relation d'aide. II importe cependant de préciser, à l'instar de Cohen (1998), que ces positions ne constituent pas des catégories distinctes d'intervenants, mais plutôt des repères sur un continuum. Voici comment nous illustrons ce continuum ainsi que les éléments qui caractérisent chacune des positions que peuvent adopter les intervenants qui travaillent en protection de la jeunesse. 


\section{Tableau 3}

\section{Objectifs poursuivis par l'intervention et principes sous-jacents}

\begin{tabular}{|c|c|c|}
\hline Contrôle & Enseignement & Empowerment \\
\hline $\begin{array}{l}\text { } \text { Seul l'intervenant } \\
\text { détient du pouvoir } \\
\text { dans l'intervention. }\end{array}$ & $\begin{array}{l}\diamond \text { L'intervenant et le } \\
\text { client s'entendent } \\
\text { sur l'inégalité du } \\
\text { pouvoir. }\end{array}$ & $\begin{array}{l}\diamond \text { Le pouvoir est } \\
\text { partagé également } \\
\text { entre le client et } \\
\text { l'intervenant. }\end{array}$ \\
\hline $\begin{array}{l}\diamond \text { Le parent doit se } \\
\text { conformer à } \\
\text { l'autorité organisa- } \\
\text { tionnelle que } \\
\text { représente } \\
\text { l'intervenant. }\end{array}$ & $\begin{array}{l}\diamond \text { L'intervenant pos- } \\
\text { sède les connais- } \\
\text { sances et l'expé- } \\
\text { rience nécessaires } \\
\text { pour conseiller le } \\
\text { parent et l'aider à } \\
\text { développer ses } \\
\text { compétences. }\end{array}$ & $\begin{array}{l}\text { \& Il y a reconnais- } \\
\text { sance et complé- } \\
\text { mentarité des } \\
\text { compétences du } \\
\text { client et de l'inter- } \\
\text { venant. }\end{array}$ \\
\hline $\begin{array}{l}\checkmark \text { Le parent est peu } \\
\text { ou pas impliqué } \\
\text { dans les décisions. }\end{array}$ & $\begin{array}{l}\text { \& Le parent est } \\
\quad \text { impliqué dans cer- } \\
\text { taines décisions. }\end{array}$ & $\begin{array}{l}\diamond \text { Le parent est impli- } \\
\text { qué dans toutes } \\
\text { les décisions. } \\
\text { L'orientation qu'il } \\
\text { souhaite donner à } \\
\text { l'intervention est } \\
\text { respectée. }\end{array}$ \\
\hline
\end{tabular}

Il importe de ne pas perdre de vue le caractère exploratoire de cette recherche. Des études qui opérationnalisent de manière plus spécifique le concept d'empowerment sont essentielles afin d'évaluer l'ancrage de cette philosophie dans l'action. De plus et comme d'autres auteurs (Page, Poertner et Lindbloom, 1995; Unger et Nelson, 1990), nous adhérons à la conviction qu'il faut encore mener plusieurs études évaluatives rigoureuses avant de pouvoir tabler sur l'efficacité des pratiques d'empowerment, et ce, particulièrement dans un contexte d'intervention exceptionnel comme l'est celui de la protection de la jeunesse. Plus spécifiquement, il se révèle nécessaire d'identifier les aspects des inter- 
ventions axées sur les compétences qui apportent des changements positifs chez ces familles. En ce sens, il est permis de faire l'hypothèse que l'écologie propre aux services offerts en vertu de la Loi sur la protection de la jeunesse comporte des facettes qui, a priori, peuvent sembler incompatibles avec une perspective d'intervention destinée à développer le pouvoir d'agir des personnes. Tout le défi consiste donc à poursuivre les efforts visant à mieux équilibrer les forces en présence afin que toutes convergent vers le développement positif des jeunes.

Marie-Christine SAINT-JACQUES

Professeure

École de service social

Université Laval

Geneviève LESSARD

Professionnelle de recherche Centre jeunesse de Québec Institut universitaire sur les jeunes en difficulté

Sylvie DRAPEAU Professeure Département des fondements et des pratiques en éducation

Université Laval

\author{
André BEAUDOIN \\ Professeur \\ École de service social \\ Université Laval
}

Marie-Christine Saint-Jacques, Sylvie Drapeau et André Beaudoin sont chercheurs au sein de l'équipe Jeunes et familles en transition du Centre de recherche sur les services communautaires de l'Université Laval et chercheurs à l'Institut universitaire sur les jeunes en difficulté - Centre jeunesse de Québec. 


\section{Références bibliographiques}

ASSOCIATION DES CENTRES JEUNESSE DU QUÉBEC (1996). Vers une plus grande implication des parents et de leur jeune - Une réflexion à faire, des outils pour y parvenir, document non paginé.

BARDIN, L. (1986). L'analyse de contenu, Paris, Presses universitaires de France, $233 \mathrm{p}$.

BERG, I.K. (1996). Services axés sur la famille, Québec, Edisem, 216 p.

BLYTHE, B.J., M.J. JoRdANA et S.A. Kelly (1991). " Family Preservation with Substance Abusing Families: Help that Works », Children and Family Services Quarterly, vol. 14, n 3, p. 12-13.

BOUSHEL, M. et M. LEBACQ (1992). « Towards empowerment in child protection work ", Children \& Society, vol. 6, n 1, p. 38-50.

BOUTIN, G. et P. DURNING (1994). Les interventions auprès des parents : Bilan et analyse des pratiques socio-éducatives, Toulouse, Privat, $208 \mathrm{p}$.

Callahan, M. et C. LUMB (1995). "My Cheque and my Children: The long road to empowerment in child welfare », Child Welfare, vol. 74, $\mathrm{n}^{\circ}$ 3, p. $795-819$.

ChARPENTIER, A. (1997). L'empowerment: Le défi des intervenantes et des intervenants auprès des parents de jeunes contrevenants de 12 à 15 ans dans la région d'Ottawa, mémoire de maîtrise, École de service social, Université d'Ottawa.

COHEN, M.B. (1998). "Perceptions of power in client/worker relationships », Families in Society, vol. 79, n 4, p. 433-442.

Corby, B., M. MiLlAR et L. YOUNG (1996). «Parental participation in child protection work: rethinking the rhetoric », British Journal of Social Work, vol. 26, p. 475-492.

DeLGado-GaitAN, C. (1991). "Involving parents in schools : A process of empowerment », American Journal of Education, vol. 100, $\mathrm{n}^{\circ} 1$, p. 20-46.

DiCKERSON, F.B. (1998). "Strategies that foster empowerment », Cognitive and Behavioral Practice, vol. 5, p. 255-275.

DRolet, M. (1997). «Empowerment et intervention familiale: concept paradoxal occultant parfois la pauvreté », Reflets, vol. 3, no 1, p. 55-79. 
Fraser, M.W., P.J. Pecora et D.A. HaApala (1991). Families in Crisis : The Impact of Family Preservation Services, New York, Aldine de Gruyter, 354 p.

GERRIS, J.R.M., N.M.C. VAN AS, P.M.A. WELS et J.M.A.M. JANSSENS (1998). "From parent education to family empowerment programs ", dans J.R. Lutzker, Handbook of Child Abuse Research and Treatment, New York, Plenum Press, p. 401-426.

GoYetTE, A., K. MARR et J.-A. LEWICKI (1994). "The family and community in milieu treatment: Challenging the parameters of residential treatment », Journal of Child and Youth Care, vol. 9, $n^{\circ} 4$, p. 39-50.

HEALY, K. (1998). « Participation and child protection : The importance of context », British Journal of Social Work, vol. 28, p. 897-914.

HEGAR, R.L. et J.M. HUNZEKER (1988). « Moving toward empowermentbased practice in public child welfare », Social Work, vol. $33, n^{\circ} 6$, p. 499-502.

HILDEBRANT, E. (1996). "Building community health care : A model and example from South Africa », IMAGE, vol. 28, n 2, p. 155-159.

JENSON, J.M. et J.K. WhitTAKER (1987). "Parental involvement in children's residential treatment », Children and Youth Services Review, vol. 9, p. 81-100.

JUTRAS, S. (1996). "L'appropriation. Un modèle approprié pour la promotion de la santé mentale des enfants ", Revue canadienne de santé mentale communautaire, vol. $15, n^{\circ} 2$, p. 123-142.

Koren, P.F., N. DeChILlo et B.J. FRIESEN (1992). « Measuring empowerment in families whose children have emotional disabilities: A brief questionnaire », Rehabilitation Psychology, vol. 37, $\mathrm{n}^{\circ} 4$, p. 305-321.

KRUEGER, R.A. (1994). Focus groups: A practical guide for applied research, Thousand Oaks, Sage Publications, $255 \mathrm{p}$.

Laflamme, M.K., T. Lavoie, R. Desgagné et D. Marion (1994). Programme AERONEF, $3^{\mathrm{e}}$ édition, Québec, Les Centres jeunesse de Québec, $64 \mathrm{p}$.

Le Bossé, Y. et M. LaVAlLÉE (1993). «Empowerment et psychologie communautaire - Aperçu historique et perspectives d'avenir », Les Cahiers internationaux de psychologie sociale, vol. 18, p. 7-20. 
LE BossÉ, Y. (1996). " Empowerment et pratiques sociales : illustration du potentiel d'une utopie prise au sérieux », Nouvelles pratiques sociales, vol. $9, \mathrm{n}^{\circ} 1$, p. 127-145.

LE BossÉ, Y. (1999). Le pouvoir d'agir : potentiel intégrateur pour les pratiques sociales. Conférence présentée le 17 mars 1999 dans le cadre des conférences scientifiques du Centre de recherche sur les services communautaires, Université Laval.

LEON, A.M. (1999). « Family support model : Integrating service delivery in the twenty-first century ». Families in Society, vol. 80, p. 14-24.

L'ÉCUYER, R. (1990). Méthodologie de l'analyse développementale de contenu, Sillery, Presses de l'Université du Québec, 472 p.

LeUnG, P., K.F.M. Cheung et K.M. SteVenson (1994). "A strengths approach to ethnically sensitive practice for child protective service workers », Child Welfare, vol. 73, n 6, p. 707-721.

MaRTone, W.P., G.F Kemp et S.J. Pearson (1989). " The continuum of parental involvement in residential treatment: Engagement Participation - Empowerment - Discharge », Residential Treatment for Children and Youth, vol. 6, n 3, p. 11-37.

MASSON, J. et C. HARRISON (1994). "Working in partnership with "lost" parents : issues of theory and practice ", Adoption \& Fostering, vol. $18, n^{\circ} 1$, p. $40-44$.

McCannell Saulnier, K. et C. Rowland (1985). "Missing links : An empirical investigation of network variables in high-risk families ", Family Relations, vol. 34, p. 557-560.

MCWHIRTER, E.H. (1998). "An empowerment model of counsellor education ", Revue canadienne de counseling, vol. 32, n 1, p. 12-26.

PAge, M., J. Poertner et R. Lindbloom (1995). "Promoting the preschooler's chances for success : A program efficacy review for behaviorally disordered or emotionally troubled children », Early Child Development Care, vol. 106, p. 167-176.

PAQUET, G. (1997). Transfert des acquis - Alternance entre le milieu de réadaptation et le milieu naturel, Québec, Le Centre jeunesse de Québec, $42 \mathrm{p}$.

PARSONS, R.J. (1991). « Empowerment : purpose and practice principles in social work », Social Work with Groups, vol. 14, n 2 , p. 7-21.

Pecora, P.J., J.K. WitTAKer et A.N. MAluccio (1992). The Child Welfare Challenge: Policy, Practice and Research, New York, Aldine de Gruyter, 526 p. 
PeRKINS, D.D. et M.A. ZIMMERMAN (1995). " Empowerment, theory, research and application », American Journal of Community Psychology, vol. $23, n^{\circ} 5$, p. 569-579.

PRESTON-SHOOT, M. (1992). «On empowerment, partnership, and authority in groupwork practice: A training contribution », Groupwork, vol. 5, n 2 , p. 5-30.

Richardson, G., B. Galaway, J. Hudson, R. Nutter et M. Hill (1995). « Birth parent participation in treatment foster care programs in North America and the United Kingdom », dans J. Hudson et B. Galaway (dir.), Child Welfare in Canada. Research and Policies Implications, Toronto, Thompson Educational Publishing, p. 219-232.

RossI, P.H. (1992). "Assessing family preservation programs ", Children and Youth Services Review, vol. 14, p. 77-97.

RutMAN, L. (1982). "Planification d'une étude évaluative », dans R. Lecomte et L. Rutman (dir.), Introduction aux méthodes de recherche évaluative, Québec, Les Presses de l'Université Laval, p. 23-46.

SCHATZ, M.S. et W. BANE (1991). "Empowering the parents of children in substitute care : a training model », Child Welfare, vol. 70, n 6, p. 665678.

Schuerman, J.R., T.L. RzePNiCKI et J.H. LitTell (1994). Putting family first : an experiment in family preservation, New York, Aldine de Gruyter, $313 \mathrm{p}$.

SimARD, G. (1989). Animer, planifier et évaluer l'action. La méthode du focus group, Laval, Mondia, $102 \mathrm{p}$.

SIMARD, M., J. VACHON et M. MOISAN (1993). "Les difficultés de la réinsertion familiale des enfants placés ", Apprentissage et Socialisation, vol. 16, $\mathrm{n}^{\circ}$ 3, p. 241-252.

STARK, W. (1992). « Empowerment and social change : health promotion within the healthy cities project of WHO-steps toward a participative prevention program », G.W. Albee, L.A. Bond, T.V.C. Monsey (dir.), Improving Children's Lives: Global Perspectives on Prevention, Thousand Oaks, Sage Publications, 398 p.

Striefel, S., M. RoBinson et P. TRUHN (1998). "Dealing with child abuse and neglect within a comprehensive Family-Support-Program », dans J.R. Lutzker, Handbook of Child Abuse Research and Treatment, New York, Plenum Press, p. 267-289.

UNGER, D.G. et P. NELSON (1990). «Evaluating community-based parenting support programs : Successes and lessons learned from 
empowering parents of adolescents ", The Journal of Applied Social Sciences, vol. $15, \mathrm{n}^{\circ} 1$, p. 125-152.

Webster-StRATtON, C. (1998). «Parent training with low-income families: promoting parental engagement through a collaborative approach », dans J.R. Lutzker, Handbook of Child Abuse Research and Treatment, New York, Plenum Press, p. 183-210.

WHITTAKER, J. (1990). Reaching high-risk families: intensive family preservation in human services, New York, Aldine de Gruyter, 206 p. 